\title{
Antigenicity analysis and molecular characterization of two outer membrane proteins of Vibrio alginolyticus strain VA2 as vaccine candidates in tiger grouper culture
}

\begin{abstract}
Vibriosis is one of the most prevalent fish diseases caused by bacteria from the genus Vibrio. An effective method to counter Vibriosis is vaccination which increases the resistance of the immune system to subsequent pathogen infection. In this study, Vibrio alginolyticus strain VA2 isolated from infected tiger groupers was selected for determination of the antigenicity and molecular characterization of its Outer Membrane Proteins (OMPs). Protein profiling of OmpK and OmpW were evaluated by sodium dodecyl sulfate polyacrylamide gel electrophoresis (SDS-PAGE) while their antigenicity were tested by Western Blot. Results of the Western Blot assay revealed three antigenic OMP bands about 23, 31 and $34 \mathrm{kDa}$. Sequencing results showed that the full length of the target genes OmpK and OmpW of V. alginolyticus strain VA2 were 846 and 642 bp, respectively. Multiple alignment analysis showed that both regions of OmpK and OmpW were highly conserved among Vibrio species, thus are good candidates for vaccine development. Phylogenetic analysis of OmpK and OmpW showed that most branches in which they were grouped with had bootstrap values higher than $50 \%$, indicating high confidence level of the branches. The antigenic sites of OmpK and OmpW proteins were predicted which were 34 and 27, respectively, thus are good candidates for vaccine development.
\end{abstract}

Keywords: Antigenic; Conserved protein; Vaccine; Antigenicity; Cloning; OmpK; OmpW 